\title{
Zeros of the Hankel Function of Real Order and of Its Derivative
}

\author{
By Andrés Cruz and Javier Sesma
}

\begin{abstract}
The trajectories followed in the complex plane by all the zeros of the Hankel function and those of its derivative, when the order varies continuously along real values, are discussed.
\end{abstract}

1. Introduction. Many physical problems require a good knowledge of the location of zeros of the Hankel function and/or those of its derivative. For instance, the trajectories of the zeros of $H_{\nu}^{(1)}(z)$, for varying real order $\nu$, are the $k$-trajectories of the $S$-matrix singularities for quantum scattering by a hard sphere. Also, the zeros of $H_{\nu}^{(1)}(z)$ and $(d / d z) H_{\nu}^{(1)}(z)$ give, respectively, the poles and zeros of the logarithmic derivative of the external Schrödinger wave function in a short-range potential, which should match, at the edge of the potential, with the logarithmic derivative of the internal wave function.

Information provided by classical treatises [7], [4] on special functions about the zeros of $H_{\nu}^{(1)}(z)$ and $(d / d z) H_{\nu}^{(1)}(z)$ is rather insufficient. A more recent updated revision of the topic has been published by Luke [6]. In the case of integer order, $\nu=n$, two types of zeros of $H_{\nu}^{(1)}(z)$ or of $(d / d z) H_{\nu}^{(1)}(z)$ are found [1, pp. 373-374], [3] (in the principal Riemann sheet, $|\arg z| \leqslant \pi$ ):

(1) An infinite number of zeros for $|\operatorname{Re} z|>n$ just below the negative real semiaxis.

(2) A group of $n$ zeros for $|\operatorname{Re} z|<n$ which lie along the lower half of the boundary of an eye-shaped domain around $z=0$.

Our interest in this paper is on the trajectories followed by those zeros as the order varies continuously along real values. Trajectories of this kind, connecting secondtype zeros, are shown in [5]. We discuss the trajectories described by all the zeros of $H_{\nu}^{(1)}(z)$ and of $(d / d z) H_{\nu}^{(1)}(z)$ in Sections 2 and 3, respectively. It will turn out from our study that the distinction between the two types of zeros mentioned above is rather artificial, since all first-type zeros become second-type as $\nu$ increases.

In view of the well-known relations [1, Eq. 9.1.6]

$$
H_{-\nu}^{(1)}(z)=\exp (i \pi \nu) H_{\nu}^{(1)}(z), \quad H_{-\nu}^{(1) \prime}(z)=\exp (i \pi \nu) H_{\nu}^{(1) \prime}(z)
$$

we need to consider only nonnegative values of $\nu$ in our discussion.

Received December 4, 1980; revised November 3, 1981 and February 2, 1982. 1980 Mathematics Subject Classification. Primary 33A40; Secondary 65H05, 81F05.

Key words and phrases. Hankel function, derivative of the Hankel function, zeros. 
2. Zeros of $H_{\nu}^{(1)}(z)$. We have used the "steepest descent" method to find the solutions of

$$
H_{\nu}^{(1)}(z)=0
$$

The Hankel function was expressed in terms of Bessel functions, which were computed by means of their ascending series expansion. Double precision was used in the summation of the series. The order $\nu$ was considered as a parameter; small variations of it allowed us to obtain the trajectories of the zeros of $H_{\nu}^{(1)}(z)$ shown in Figure 1.

The trajectories start from the zeros of $H_{0}^{(1)}(z)$. All zeros, accurate to $10 \mathrm{D}$, of $H_{0}^{(1)}(z)$ for $|z|<158$ were obtained by Döring [3]. We shall consider these zeros, $z_{s}$, labelled by an index $s=1,2, \ldots$ increasing with the absolute value of $z_{s}$. The same label will be used for the corresponding trajectory. As $\nu$ increases, all zeros move upwards, almost vertically, approaching the cut existing along the negative real semiaxis. At $\nu=1 / 3$ the zeros cross the cut and go into the Riemann sheet $-3 \pi \leqslant \arg z \leqslant-\pi$. They continue their ascending motion and go to infinity as $\nu$ tends to $1 / 2$ from below, along the asymptotes $x=(-s+1 / 4) \pi$. As $\nu$ crosses the value $1 / 2$, all zeros make a discontinuous jump by $\Delta x=\pi / 2$. As $\nu$ increases, the zeros come from infinity along the asymptotes $x=(-s+3 / 4) \pi$. Their nearly vertical motion causes them to cross the cut at $\nu=2 / 3$, coming back in this way to the principal Riemann sheet. They continue, on this sheet, moving downwards until the order takes the value $\nu=1$. The positions of the zeros of $H_{1}^{(1)}(z)$, accurate to 10D, have been given by Döring [3].

The first zero, $z_{1}(\nu)$, that for $\nu=0$ had been considered a first-type zero according to the classification of the zeros in the two types mentioned in Section 1, is of the second type for $\nu=1$. Analogously, every zero $z_{s}(\nu)$, that should be considered a first-type zero for $\nu<s$, becomes a second-type one for $\nu \geqslant s$.

The behavior of the first-type zeros for $\nu$ in the interval $1 \leqslant \nu \leqslant 2$ or, in general, in the range $n \leqslant \nu \leqslant n+1$ is quite similar to that shown in the interval $0 \leqslant \nu \leqslant 1$. As $\nu$ increases, the zeros move upwards, cross the cut at $\nu=n+1 / 3$ and, as $\nu$ tends to $n+1 / 2$, go to infinity in the Riemann sheet $-3 \pi \leqslant \arg z \leqslant-\pi$ along the asymptotes $x=(-s+n / 2+1 / 4) \pi$, where $s>\nu$. They jump by $\Delta x=\pi / 2$ as $\nu$ passes $n+1 / 2$ and go down, along the asymptotes $x=(-s+n / 2+3 / 4) \pi$, towards the cut, which they cross at $\nu=n+2 / 3$. They continue moving downwards in the principal Riemann sheet until they stop their descending motion near $\nu=n+1$. The second-type zeros $\left(z_{s}, s \leqslant n\right)$, instead, move downwards and towards the right, following the trajectories shown in [5]. They cross the negative imaginary semiaxis at $\nu=2 s-1 / 2[1$, p. 441] and go to infinity in the fourth quadrant of the $z$ plane as $\nu$ tends to infinity. As is well known, [1, p. 441], the positions of these zeros (for different values of $s$ ) at half-integer values of $\nu, \nu=n+1 / 2$, are symmetrical with respect to the imaginary axis.

It is not difficult to understand why the zeros cross the cut precisely for $\nu=n+1 / 3$ and $\nu=n+2 / 3$. Equation (2.1) is equivalent to the condition (for $\nu$ noninteger) 


$$
\begin{aligned}
\sum_{r=0}^{\infty}\left(-z^{2} / 4\right)^{r} / r !(-\nu)(-\nu+1) & \cdots(-\nu+r) \\
& -G_{\nu}(z) \sum_{r=0}^{\infty}\left(-z^{2} / 4\right)^{r} / r ! \nu(\nu+1) \cdots(\nu+r)=0,
\end{aligned}
$$

where we have denoted

$$
G_{\nu}(z)=\exp (-i \nu \pi)\left(z^{2} / 4\right)^{\nu} \Gamma(-\nu) / \Gamma(\nu) .
$$

For $z$ on the cut, both series in (2.2) are real. So, solutions $z_{s}$ of (2.2) with $\arg z_{s}=-\pi$ can exist only if $G_{\nu}\left(z_{s}\right)$ becomes real. This happens obviously if and only if $3 \nu$ is an integer, $\nu$ noninteger.

Now, let us examine more carefully the behavior of the first-type zeros as $\nu$ approaches a half-integer, $\nu \simeq n+1 / 2$. Using the analytic continuation formula [ 1 , Eq. 9.1.37] for the Hankel function, we can write

$$
\begin{aligned}
H_{\nu}^{(1)}(z \exp (-i 2 \pi))= & \left\{4 \cos ^{2}(\nu \pi)-1\right\} H_{\nu}^{(1)}(z) \\
& +2 \cos (\nu \pi) \exp (-i \nu \pi) H_{\nu}^{(2)}(z),
\end{aligned}
$$

where $z$ is meant to lie in the principal Riemann sheet. Bearing in mind the fact that $|z| \rightarrow \infty$, the right-hand side of (2.4) can be approximated by using the asymptotic forms [1, Eqs. 9.2.3 and 9.2.4] of the Hankel functions to obtain, retaining only leading terms,

$$
\begin{aligned}
& H_{\nu}^{(1)}(z \exp (-i 2 \pi)) \\
& \simeq\left(\frac{2}{\pi z}\right)^{1 / 2}\left(\left\{4 \cos ^{2}(\nu \pi)-1\right\} \exp \{i(z-\nu \pi / 2-\pi / 4)\}\right. \\
& \quad+2 \cos (\nu \pi) \exp (-i \nu \pi) \exp \{-i(z-\nu \pi / 2-\pi / 4)\}) .
\end{aligned}
$$

The zeros of $H_{\nu}^{(1)}(z)$ are, therefore, given approximately by the roots of

$$
\exp \{-i 2(z-\pi / 4)\}=\left\{1-4 \cos ^{2}(\nu \pi)\right\} / 2 \cos (\nu \pi) .
$$

Denoting by $x_{s}$ and $y_{s}$ the real and imaginary parts of $z_{s}$, we obtain from (2.6)

$\left(2.7\right.$, a) $\quad x_{s} \simeq \pi / 4-k \pi-(1 / 2) \arg \left(\left\{1-4 \cos ^{2}(\nu \pi)\right\} / 2 \cos (\nu \pi)\right), \quad k$ integer,

$(2.7, \mathrm{~b}) \quad y_{s} \simeq(1 / 2) \log \left|\left\{1-4 \cos ^{2}(\nu \pi)\right\} / 2 \cos (\nu \pi)\right|$.

It can be seen in $(2.7, a)$ that $x_{s}$ changes discontinuously by $\pi / 2$ whenever $\nu$ increases through a half-integer value. Equation $(2.7, \mathrm{~b})$ confirms that, as $\nu$ tends to $n+1 / 2$, all zeros go to infinity, their imaginary parts being approximately independent of the label $s$.

To end this analysis of the zeros of $H_{\nu}^{(1)}(z)$ it remains only to consider their behavior as the order $\nu$ tends to infinity. Cochran [2] has discussed the zeros of the Hankel function, as function of its order, giving an approximate expression valid for large values of the variable. From that expression it is easy to obtain, for large $\nu$,

$$
\begin{aligned}
z_{s}= & \nu-2^{-1 / 3} \exp (-i 2 \pi / 3) a_{s} \nu^{1 / 3} \\
& +(3 / 10) 2^{-2 / 3} \exp (-i 4 \pi / 3) a_{s}^{2} \nu^{-1 / 3}+O\left(\nu^{-1}\right),
\end{aligned}
$$

where $a_{s}$ denotes the $s$ th one among the zeros of the Airy function of the first kind, which are all negative real $[1$, p. 478]. 


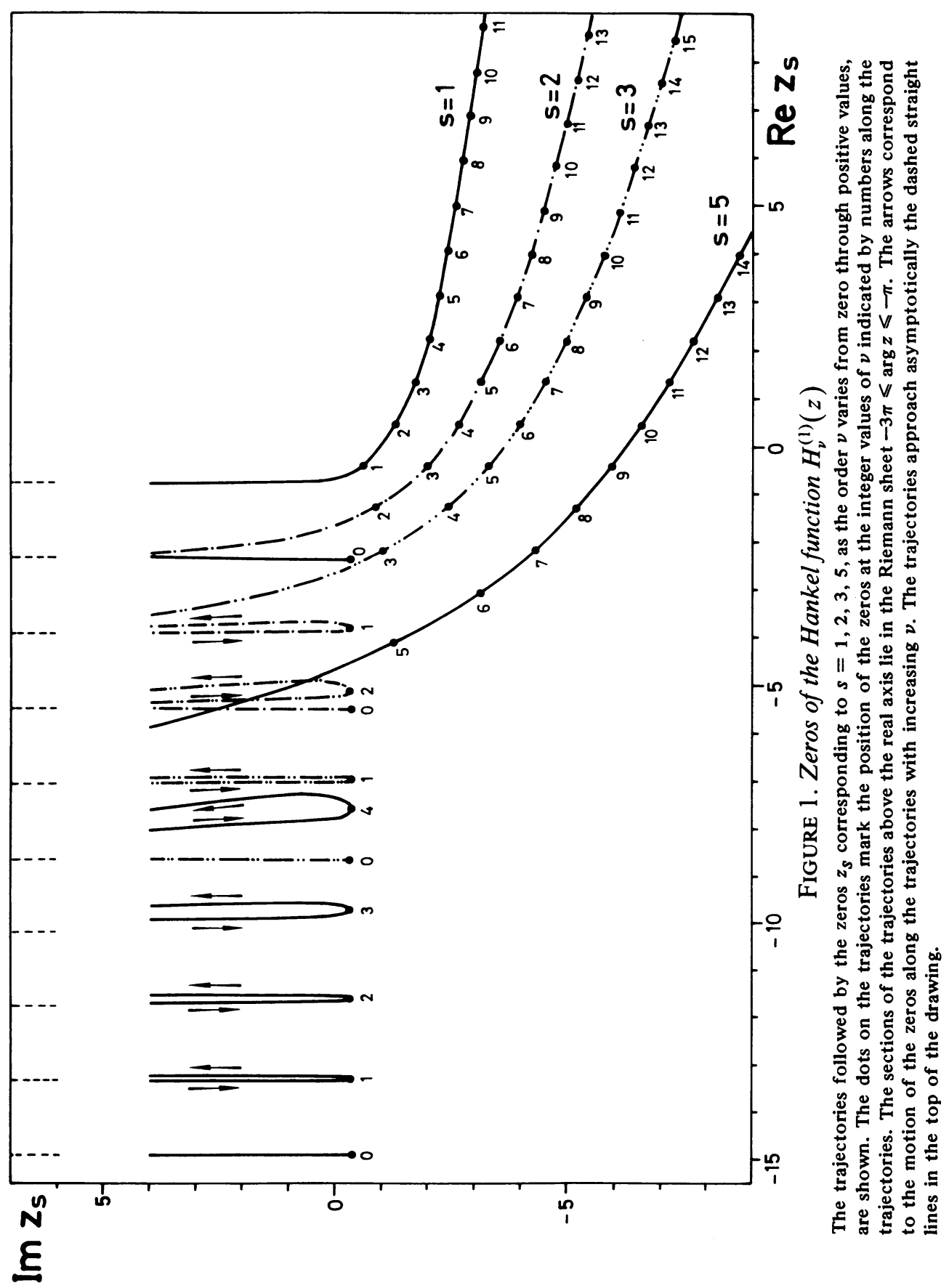




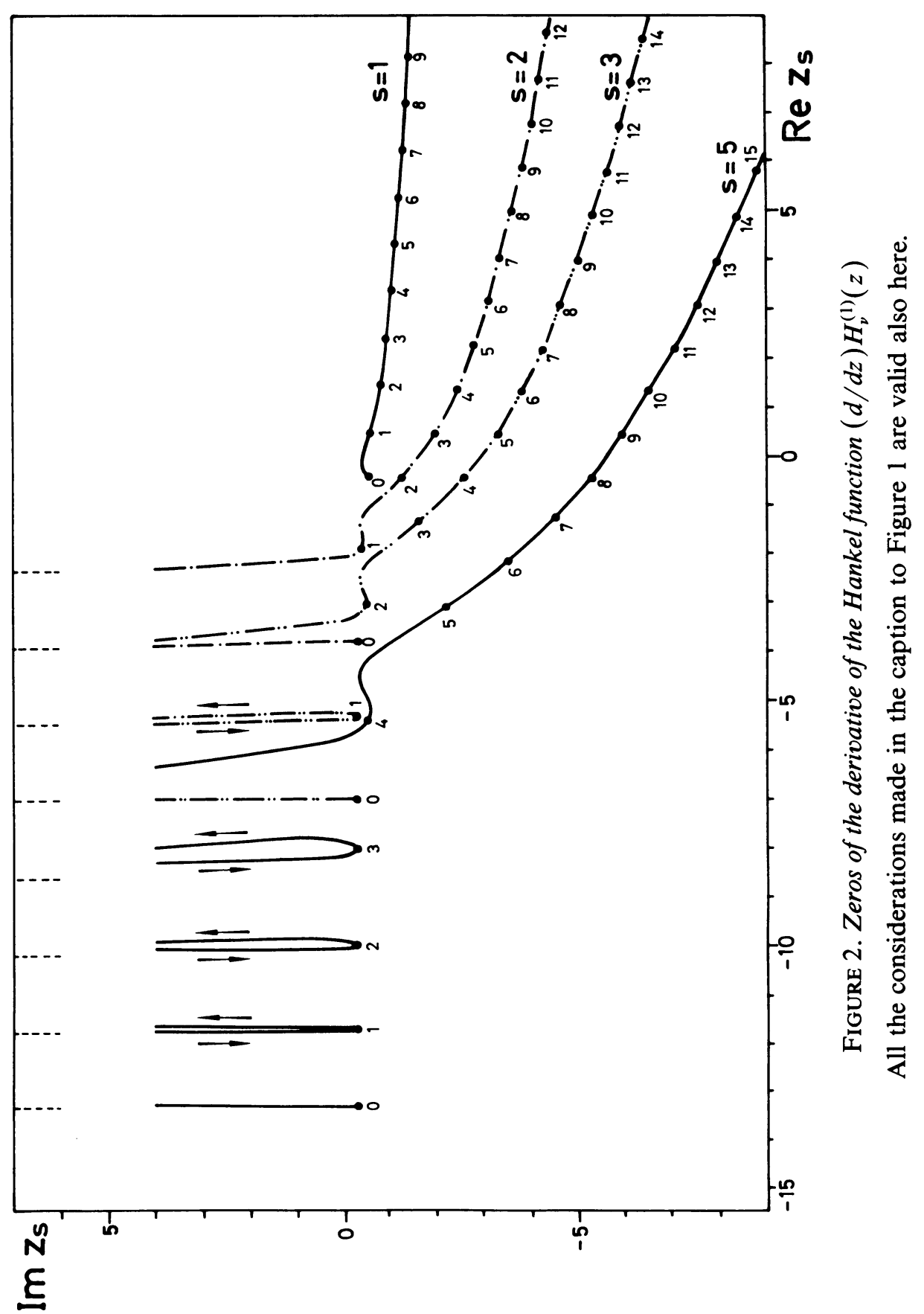


3. Zeros of $(d / d z) H_{\nu}^{(1)}(z)$. In the solution of the equation

$$
(d / d z) H_{\nu}^{(1)}(z)=0
$$

we have followed a numerical procedure entirely similar to that described in Section 2. Our results are shown in Figure 2.

The starting points, for $\nu=0$, of the trajectories are the zeros of $H_{1}^{(1)}(z)$, given in [3]. The behavior of the solutions of (3.1) as $\nu$ varies from $n$ to $n+1$ is very similar to that of the zeros of $H_{\nu}^{(1)}(z)$. All first-type zeros, except that of smallest absolute value, move vertically towards the cut, cross it at $\nu=n+1 / 3$ and tend to infinity, in the Riemann sheet $-3 \pi \leqslant \arg z \leqslant-\pi$, along the asymptotes $x=$ $(-s+n / 2+3 / 4) \pi$, with $s>n+1$, as $\nu$ tends to $n+1 / 2$. They jump by $\Delta x=$ $\pi / 2$ at $\nu=n+1 / 2$ and go down along $x=(-s+n / 2+5 / 4) \pi$ as $\nu$ increases further. They cross the cut at $\nu=n+2 / 3$ and move downwards until they stop the descending motion near $\nu=n+1$. The smallest first-type zero moves, as $\nu$ increases from $n$, upwards and to the right, makes a small bump and becomes a second-type zero. All second-type zeros go downwards and towards the right, crossing the imaginary axis at $\nu=2 s-3 / 2[1, \mathrm{p}$. 441] and going to infinity in the fourth quadrant as $\nu$ tends to infinity.

The explanation of the fact that the zeros cross the cut at $\nu=n+1 / 3$ and $\nu=n+2 / 3$ and of their behavior as $\nu$ approaches $n+1 / 2$ runs along the same lines as in Section 2. The large $\nu$ behavior can be obtained from [2]. It turns out

$$
\begin{aligned}
z_{s}= & \nu-2^{-1 / 3} \exp (-i 2 \pi / 3) a_{s}^{\prime} \nu^{1 / 3} \\
& +2^{-2 / 3} \exp (-i 4 \pi / 3)\left(3 a_{s}^{\prime 2} / 10+1 / 5 a_{s}^{\prime}\right) \nu^{-1 / 3}+O\left(\nu^{-1}\right)
\end{aligned}
$$

where $a_{s}^{\prime}$ denotes the $s$ th stationary value of the Airy function.

Appendix. It has been mentioned in Section 2 that the trajectories of the first-type zeros of $H_{\nu}^{(1)}(z)$ in the complex $z$ plane, as $\nu$ varies along real values, present relative minima for nearly integer values of $\nu$. It can be proven that such minima do not occur exactly at integer $\nu$, the case $\nu=0$ being excepted. By differentiating (2.1), one obtains for the slope of a trajectory at a given point

$$
d z / d \nu=-\left(\partial H_{\nu}^{(1)}(z) / \partial \nu\right) /\left(\partial H_{\nu}^{(1)}(z) / \partial z\right),
$$

where it is understood that $z$ and $\nu$ in the right-hand side take the values corresponding to that point of the trajectory. By expressing the derivatives in (A.1) in terms of the Hankel functions [1, Eqs. 9.1.27, 9.1.66-68] and bearing in mind that (2.1) is satisfied, one obtains in the case of integer $\nu$

$$
\begin{aligned}
& \nu=0, \quad \frac{d z}{d \nu}=0, \\
& \nu=n \neq 0, \quad \frac{d z}{d \nu}=-\frac{n !(2 / z)^{n}}{2 H_{n-1}^{(1)}(z)} \sum_{k=0}^{n-1} \frac{(z / 2)^{k} H_{k}^{(1)}(z)}{(n-k) k !} .
\end{aligned}
$$

By using again the recurrence relations [1, Eq. 9.1.27] for the Hankel functions and (2.1), it is easy to see that the right-hand side of $(\mathrm{A} .2, \mathrm{~b})$ reduces to an odd 
polynomial of degree $2 n-1$ in $z^{-1}$. For the lowest values of $\nu, d z / d \nu$ turns out to be

$$
\begin{aligned}
& \nu=0, \quad d z / d \nu=0, \\
& \nu=1, \quad d z / d \nu=-1 / z, \\
& \nu=2, \quad d z / d \nu=-(1 / z)\left(2+4 / z^{2}\right), \\
& \nu=3, \quad d z / d \nu=-(1 / z)\left(3+16 / z^{2}+64 / z^{4}\right),
\end{aligned}
$$

where $z$ is to be replaced by the corresponding zero of $H_{n}^{(1)}(z)$. A relative minimum of the trajectory at a given point should be recognized by a vanishing imaginary part of $d z / d \nu$ at that point. Obviously, the zeros at $\nu=n \neq 0$ are not relative minima of the trajectories.

A similar conclusion can be obtained for the relative minima of the trajectories of the zeros of $(d / d z) H_{\nu}^{(1)}(z)$, mentioned in Section 3. Analogously to (A.2), one obtains

$$
\begin{aligned}
(\mathrm{A} .3, \mathrm{a}) \quad \nu=0, \quad \frac{d z}{d \nu}= & 0, \\
\nu=n \neq 0, \quad \frac{d z}{d \nu}= & \frac{n !(2 / z)^{n-1}}{4\left(1-n^{2} / z^{2}\right) H_{n}^{(1)}(z)} \\
(\mathrm{A} .3, \mathrm{~b}) \quad & \times \sum_{k=0}^{n-1} \frac{(z / 2)^{k}}{(n-k) k !}\left[(2 k-n) H_{k}^{(1)}(z)-z H_{k+1}^{(1)}(z)\right] .
\end{aligned}
$$

The right-hand side of $(\mathrm{A} .3, \mathrm{~b})$ can be reduced to the quotient of an odd polynomial of degree $2 n+1$ divided by an even polynomial of the second degree, both in $z^{-1}$. For the lowest values of $\nu, d z / d \nu$ becomes

$$
\begin{aligned}
& \nu=0, \quad d z / d \nu=0, \\
& \nu=1, \quad d z / d \nu=-(1 / z)\left(1+1 / z^{2}\right) /\left(1-1 / z^{2}\right) \text {, } \\
& \nu=2, \quad d z / d \nu=-(1 / z)\left(2+16 / z^{4}\right) /\left(1-4 / z^{2}\right) \text {, } \\
& \nu=3, \quad d z / d \nu=-(1 / z)\left(3-5 / z^{2}+48 / z^{4}+576 / z^{6}\right) /\left(1-9 / z^{2}\right) .
\end{aligned}
$$

Acknowledgements. The authors would like to thank the referee for his criticisms and suggestions. Financial support of Instituto de Estudios Nucleares is also acknowledged.

Departamento de Fisica Teorica

Facultad de Ciencias

Universidad de Zaragoza

Zaragoza, Spain

1. M. Abramowitz \& I. A. Stegun (Editors), Handbook of Mathematical Functions, Dover, New York, 1965.

2. J. A. Cochran, “The zeros of Hankel functions as functions of their order," Numer. Math., v. 7 , 1965, pp. 238-250.

3. B. Döring, "Complex zeros of cylinder functions," Math. Comp., v. 20, 1966, pp. 215-222.

4. A. Erdelyi, W. Magnus, F. Oberhettinger \& F. G. Tricomi, Higher Transcendental Functions, vol. 2, McGraw-Hill, New York, 1953, p. 62.

5. E. JAHNKe, F. EMdE \& F. LöSCH, Tables of Higher Functions, McGraw-Hill, New York, 1960. p. 229.

6. Y. L. Luke, Mathematical Functions and their Approximations, Academic Press, New York, 1975.

7. G. N. Watson, $A$ Treatise on the Theory of Bessel Functions, 2nd ed., Cambridge Univ. Press, New York, 1958, Chapter XV. 